\title{
Rigorous Construction of Planar Diagram Field Theories in Four Dimensional Euclidean Space
}

\author{
Gerard 't Hooft \\ Institute for Theoretical Physics, Princetonplein 5, P.O. Box 80.006, NL-3508 TA Utrecht, \\ The Netherlands
}

\begin{abstract}
Asymptotically free quantum field theories with planar Feynman diagrams [such as $\mathrm{SU}(\infty)$ gauge theory] are considered in 4 dimensional Euclidean space. It is shown that if all particles involved have non-vanishing masses and if the coupling constant(s) $\lambda$ (or $\left.g^{2}\right)$ are small enough $\left(\lambda \leqq \lambda^{\text {crit }}\right)$, then an absolutely convergent procedure exists to obtain Green functions that uniquely solve the Dyson-Schwinger equations.
\end{abstract}

\section{Introduction}

In two previous papers $[1,2]$ this author explained his interest in planar diagram field theories. To date no analytic method for summing planar diagrams is known, although interesting exact features of the corresponding $N \rightarrow \infty$ field theories were displayed [3]. We now suspect that convergent calculational procedures do exist in these theories, but we are unable to prove this for the most interesting case: $\mathrm{SU}(\infty) \mathrm{QCD}$. The problem is the fact that the gluons are massless. In this paper we consider a closely related set of theories. The Feynman rules may be as in planar QCD, except that we take all particles involved to be massive, and the coupling constant must be smaller than a certain limit. Furthermore, our theory must be asymptotically free. Since we only look at the Feynman rules and are not concerned about unitarity or positivity of the energy, the signs of the couplings can always easily be arranged in such a way that asymptotic freedom is possible. For such a theory we will construct an absolutely convergent calculational procedure. In another publication [4] we already indicated how this may imply absolute Borel summahility of the perturbation expansion of this system. Since the mass of the particles will only be needed in the very end of our argument we will also be able to draw certain conclusions about the Borel functions in massless $\mathrm{SU}(\infty)$ QCD. Further details are postponed to a future publication. There may be some optimism that $\mathrm{SU}(\infty)$ QCD may also be rigorously constructed sometime, but prospects for finite $\mathrm{SU}(N)$ theories seem to be much more remote.

Our technique will be that of [2]. There it was shown that if we sum only the ultraviolet-convergent planar diagrams then this sum has a finite radius of 
convergence ${ }^{1}$. What has to be done here is to replace the bare propagators, 3 -vertices and 4-vertices of [2] by dressed propagator and vertex functions. It is easy to convince oneself that then all (planar) Feynman graphs are generated. The planar diagram expansion of [2] then is called "skeleton expansion," and an important ingredient of our present argument is that the transition to dressed propagator- and vertex functions still leaves the skeleton expansion convergent, provided that the 2-, 3-, and 4-point functions fall within certain limits.

We will call the 2-, 3-, and 4-point functions the "basic Green functions," in contrast with Green functions with 5 or more external lines, which can be expanded in series of skeleton graphs ("expandable Green functions"). Inside a skeleton graph only basic Green functions occur.

As a first Ansatz (which will be justified in the end, Sect. 6), we assume that the basic Green functions are sufficiently reasonably behaved (Sect. 4). We then deduce from the field equations certain relations ("difference equations") that express the difference between basic Green functions at different external momenta in terms of expandable Green functions, for which we write down skeleton expansions.

We then solve the difference equations iteratively (Sect.6) in order to find improved expressions for the basic Green functions, in which the only unknowns are the values of these basic Green functions at their symmetry points. One can check that the ansatz in the beginning is indeed correct. After applying some Ward identities one finds as the only unknowns some floating coupling constants $g_{i}(\mu)$, as arbitrary functions of a parameter $\mu$ with dimensions of a mass. For these one can also write down the difference equations which then take the form of renormalization group equations with correction terms. One then shows that these higher order correction terms are sufficiently small as to not disturb the uniqueness of the solutions of these renormalization group equations, and that indeed non-trivial solutions exist (Sect. 8). This means that we must verify that the floating coupling constants $g(\mu)$ obtained do not exceed the radius of convergence for the skeleton expansions, and that an iterative procedure to obtain a solution converges.

For actual calculations (of the mass spectrum for instance) the procedure we sketched may not be very practical. But from its existence one will be able to prove that the technically much more convenient Borel resummation procedure for the perturbation expansion also converges and analytic properties of the Borel functions $F(z)$ may be derived [4].

\section{The Basic Green Functions}

In planar diagrams it is convenient to label external momenta not by one but by two consecutive indices:

$$
p_{i, i+1} \equiv p_{i}-p_{i+1}, \quad(i \text { cyclic }) .
$$

1 The author was informed that this result can easily be derived from the results of de Calan and Rivasseau [5], who write down more general bounds for massive, non-planar renormalized divergent graphs. Some rewriting must be done however because direct application of their main theorem gives a too divergent high-momentum behavior 
This way momentum conservation $\left(\sum_{i} p_{i, i+1}=0\right)$ is automatic. Here $p_{i}$ are defined by (2.1) up to an arbitrary overall translation in momentum space. All planar channels (the only ones in which planar theories can have resonances) then always have momenta given by the difference of two $p_{i}$. Thus in a 4-point function the $s$-channel momentum is $p_{13}=p_{1}-p_{3}$ and the $t$-channel momentum is $p_{24}=p_{2}-p_{4}$. Since the indices here refer to loops adjacent to our diagram, $p_{i}$ will be called external loop momenta, and $p_{i, i+1}$ are external line momenta.

The symmetry point (actually a line) of an amputated irreducible [6] 4-point function: $G_{4}\left(p_{1}, p_{2}, p_{3}, p_{4}\right)$ is defined by

$$
p_{12}^{2}=p_{23}^{2}=p_{34}^{2}=p_{41}^{2}=p_{13}^{2}=p_{24}^{2} \equiv p^{2},
$$

and for a 3-point function: $G_{3}\left(p_{1}, p_{2}, p_{3}\right)$,

$$
p_{12}^{2}=p_{23}^{2}=p_{31}^{2}=p^{2} \text {. }
$$

If more than one particle type is involved these functions may have indices labeling the particles in the external lines, and in gauge theories these may be Lorentz indices ${ }^{2}$. We can enumerate all possible Lorentz covariant ways in which these Lorentz indices are linked to the external momenta. This way we get a number of Lorentz-invariant structure functions at the symmetry points. These functions then still depend on one momentum-squared $p^{2}$.

For example in a pure gauge theory we have at the symmetry point

$$
\begin{aligned}
G_{3}^{\mu \nu \lambda}\left(p_{1}, p_{2}, p_{3}\right)= & i A\left(p^{2}\right)\left\{\delta_{\mu \nu} q_{\lambda}^{3}+\delta_{\nu \lambda} q_{\mu}^{1}+\delta_{\lambda \mu} q_{\nu}^{2}\right\}+i B\left(p^{2}\right) q_{\mu}^{1} q_{\nu}^{2} q_{\lambda}^{3} \\
& +i C\left(p^{2}\right)\left\{q_{\mu}^{2} q_{\nu}^{1} q_{\lambda}^{3}+q_{\mu}^{1} q_{\nu}^{3} q_{\lambda}^{2}+q_{\mu}^{3} q_{\nu}^{2} q_{\lambda}^{1}\right\},
\end{aligned}
$$

where

$$
\begin{aligned}
& q^{1}=p_{2}+p_{3}-2 p_{1}=p_{31}-p_{12}, \\
& q^{2}=p_{1}+p_{3}-2 p_{2}=p_{12}-p_{23}, \\
& q^{3}=p_{1}+p_{2}-2 p_{3}=p_{23}-p_{31} .
\end{aligned}
$$

Of course there are similar expressions for other 3- and 4-point functions.

At lowest order in the coupling constant we have:

$$
A^{0}\left(p^{2}\right)=\frac{1}{2} g ; \quad B^{0}\left(p^{2}\right)=C^{0}\left(p^{2}\right)=0 .
$$

The function $A\left(p^{2}\right)$ for the dressed vertex can be related to a running coupling constant $g(\mu)$ as follows:

$$
2 A\left(p^{2}\right)=g(\mu) Z(\mu)^{-3 / 2} ; \mu^{2}=|p|^{2},
$$

where $Z(\mu)$ is the "field renormalization factor," coming from the two-point functions:

$$
G_{2}^{\mu \nu}(p, 0)=-Z(\mu)^{-1}\left(p^{2} \delta_{\mu \nu}-p_{\mu} p_{v}\right)-\alpha(\mu)^{-1} p_{\mu} p_{v},
$$

where $\alpha(\mu)$ is an arbitrary gauge parameter.

2 Of course, the color index which runs from 1 to $N$, where $N$ tends to infinity in planar theories, was already removed by absorbing $N$ into the coupling constant [7] 
Again we keep in mind that these expressions must be generalized for the more complicated massive theories. Notice that in our formalism, $Z(\mu)$ is finite. Only renormalized, subtracted expressions are considered.

The running coupling constant(s) will satisfy a modified version of renormalization group (Gell-Mann-Low [8]) equations, which will be of the form

$$
\frac{\mu d}{d \mu} g_{i}(\mu)=-\beta_{i}^{(0)}\left(g_{j}(\mu)\right)-\beta_{i}^{(1)}\left(g_{j}(\mu)\right)+\mathcal{O}(g(\mu))^{N},
$$

where either $\beta^{(0)}$ is essentially quadratic in the $g_{j}(\mu), \beta^{(1)}$ cubic and $N=4$, or $\beta^{(0)}$ is cubic, $\beta^{(1)}$ a fifth power and $N=7$, depending on the nature of the coupling constants.

Here $\mathcal{O}(g(\mu))^{N}$ contains all higher order effects, and it will be defined later; we indicated explicitly the negative sign of the first coefficient. The coefficients $\beta^{(0)}$, $\beta^{(1)}$, and sometimes even more are known from standard perturbation theory [9]. In all but the simplest theories these coefficients are tensors. We then only consider a solution of (2.9) that converges to zero at $\mu \rightarrow \infty$ (asymptotic freedom). The functions $B$ and $C$ of Eq. (2.4) must go to zero faster than $1 / \mu^{2}$.

The solution of Eq. (2.9) has the form

$$
g_{i}(\mu)=\xi_{i}(\mu) \lambda(\mu)
$$

or

$$
g_{i}(\mu)=\xi_{i}(\mu) \sqrt{\lambda(\mu)}
$$

in the two cases mentioned before, where $|\xi|^{2}=1$ and $\xi_{i}(\mu) \rightarrow \xi_{i}$ as $\mu \rightarrow \infty$, and $\lambda$ satisfies

$$
\frac{\mu d \lambda}{d \mu}=-\beta^{0}(\mu) \lambda^{2}-\beta^{1}(\mu) \lambda^{3}+\mathcal{O}\left(\lambda^{4}\right),
$$

with definitions of the form

$$
\begin{gathered}
\beta^{0}(\mu)=(2) \xi_{i} \beta_{i}^{(0)}(\xi) \underset{\mu \rightarrow \infty}{\longrightarrow} \beta_{0} ; \\
\beta^{1}(\mu)=(2) \xi_{i} \beta_{i}^{(1)}(\xi) \underset{\mu \rightarrow \infty}{\longrightarrow} \beta_{1} ; \\
\lambda^{-1} \rightarrow \beta_{0} \log (\mu / \Lambda)+\kappa \log \log (\mu / \Lambda)+\mathcal{O}(1 / \log (\mu / \Lambda)) .
\end{gathered}
$$

Here $\xi_{i}$ is a solution of

$$
\beta_{0} \xi_{i}=\beta_{i}^{0}(\xi)
$$

and $\Lambda$ is an integration constant. There is no need for the solution given by (2.10)-(2.14) to be stable, and besides $\Lambda$ there could be more free parameters hidden in the functions $\xi_{i}(\mu)$, although we do not know of examples of such multiple parameter asymptotically free theories relevant to nature.

We have for the field renormalization factor $Z(\mu)$ an asymptotic form that usually goes like

$$
Z(\mu) \underset{\mu \rightarrow \infty}{\longrightarrow}[\log (\mu / \Lambda)]^{\sigma},
$$

where $\sigma$ is a gauge dependent coefficient, to be determined from one-loop perturbation theory. 
We consider (2.10)-(2.15) as boundary conditions for our theory. A crucial point of our argument will be to derive equations that replace (2.9) at finite $\mu$ with increasing accuracy, and to show that the boundary conditions (2.10)-(2.15) determine the solution uniquely. It will turn out that we must require inequalities of the form

$$
|\lambda(\mu)|<\lambda^{\text {crit }}
$$

for all $\mu$, where $\lambda^{\text {crit }}$ is a small positive number related to the radius of convergence derived in [2]. This forces us to consider massive theories only because otherwise $\lambda(\mu)$ necessarily becomes too large.

\section{Difference Equations for Basic Green Functions}

The Feynman rules of our set of theories must follow from a Lagrangian, as usual. From this Lagrangian one can derive Dyson-Schwinger equations. These are equations that relate Green functions to other Green functions in such a way that, formally, all Green functions should be determined by solving these equations iteratively. Now the problem with the Dyson-Schwinger equations as they are usually written is that they require subtractions of infinities. This makes iterative estimates of magnitudes of amplitudes difficult, so we decided to use altered versions, more suitable for expansions in skeleton diagrams.

For brevity we ignore the Lorentz indices and such, because those details are not of much concern to us. Let the dressed propagator be

$$
P(p)=-G_{2}^{-1}(p)
$$

and let the corresponding zeroth order expressions be indicated by adding a superscript 0 . In massive theories:

$$
P^{0}(p)=\left(p^{2}+m^{2}\right)^{-1}=-G_{2}^{0-1}(p) .
$$

Define

$$
G_{2}(p+k)-G_{2}(p)=G_{2 \mu}(p \mid k) k_{\mu}
$$

so that

$$
P(p+k)-P(k)=P(p+k) G_{2 \mu}(p \mid k) k_{\mu} P(p) .
$$

This gives us the "Feynman rule" for the difference of two dressed propagators, depicted in Fig. 1. (Note that, in this section only, $p$ and $k$ denote external line momenta, not external loop momenta.)

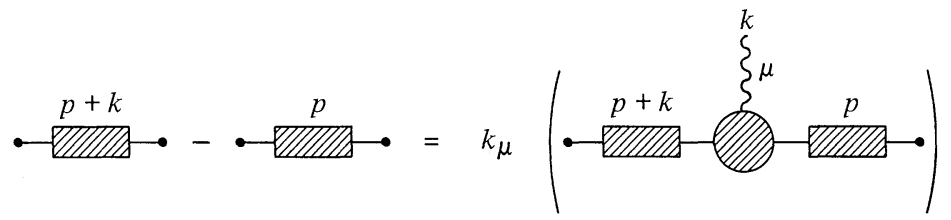

Fig. 1. Feynman rule for the difference of two dressed propagators. The 3-vertex at the right is the function $G_{2 \mu}(p \mid k)$ 


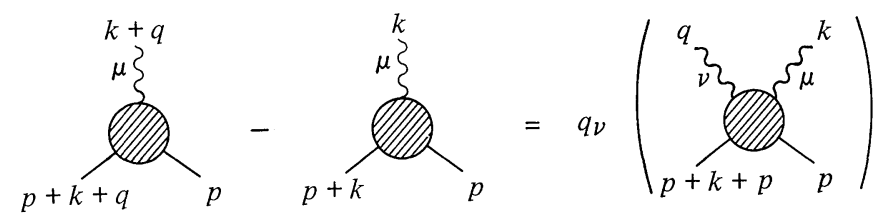

Fig. 2. Difference equation (3.6) for $G_{2 \mu}$

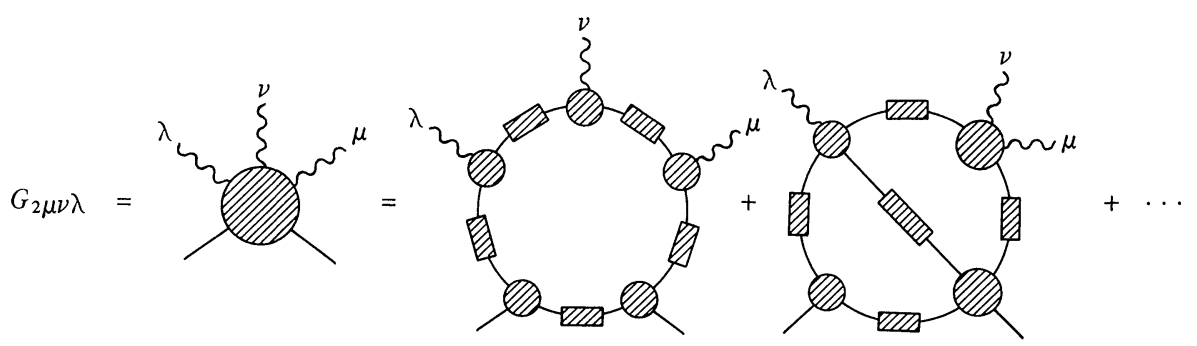

Fig. 3. Some arbitrarily chosen terms in the skeleton expansion for $G_{2 \mu \nu}$

We have also this Feynman rule for bare propagators. There $G_{2 \mu}^{0}$ follows directly from the Lagrangian:

$$
G_{2 \mu}^{0}=-2 p_{\mu}-k_{\mu}
$$

Continuing this way we define

$$
G_{2 \mu}(p \mid k+q)-G_{2 \mu}(p \mid k)=G_{2 \mu \nu}(p|k| q) q_{\nu},
$$

with

$$
G_{2 \mu \nu}^{0}=-\delta_{\mu \nu} .
$$

In Feynman graphs this is sketched in Fig. 2. Differentiating once more we get

$$
G_{2 \mu \nu}(p|k| q+r)-G_{2 \mu \nu}(p|k| q)=G_{2 \mu \nu \lambda}(p|k| q \mid r) r_{\lambda} .
$$

Of course $G_{2 \mu \nu \lambda}$ can be computed formally in perturbation expansion. Considering the corresponding Feynman graphs and substituting the expressions (3.4) and (3.6) for the bare propagators, we find a set of Feynman rules for computing $G_{\mu}, G_{\mu \nu}$ etc. directly. The bare functions $G_{2 \mu}^{0}$, etc. occur at one edge of the planar diagram. We see that the power counting rules for divergences in $G_{2 \mu \nu \lambda}$ are just as in 5-point functions in gauge theories. Since the global degree of divergence is negative we can expand in skeleton graphs. See Fig. 3, in which the blobs represent ordinary dressed propagators and dressed vertices or dressed functions $G_{\mu}$ and $G_{\mu \nu}$.

Notice that one might also need $G_{3 \mu}\left(p_{1}, p_{2} \mid k\right)$ defined by

$$
G_{3}\left(p_{1}, p_{2}+k\right)-G_{3}\left(p_{1}, p_{2}\right)=G_{3 \mu}\left(p_{1}, p_{2} \mid k\right) \cdot k_{\mu} .
$$

In short, the skeleton expansion expresses $G_{2 \mu \nu \lambda}$ but also $G_{3 \mu \nu}$ etc. in terms of the few basic functions $G_{2 \mu}, G_{2 \mu \nu}, G_{3 \mu}$ and the basic Green functions $G_{2,3,4}$. Also the function $G_{4 \mu}$, defined similarly, can thus be expressed. The corresponding Feynman rules should be clear and straightforward. 


\section{Inequalities as Ansätze}

As a starting point for an iteration procedure we must use some rough approximation to the basic (=primitively divergent) Green functions including $G_{3 \mu}, G_{2 \mu \nu}$ etc. They should be inserted in the skeleton diagrams at the right hand side of the difference equations such as Fig. 3. Then by solving Eqs. (3.8), (3.6), and (3.3) (in that order), under boundary conditions determined by the asymptotic form of the renormalization group, we find new approximations to the elementary Green functions. Our claim is that under certain conditions (to be specified) this iteration procedure converges.

The first trial must be chosen sufficiently carefully. The elementary Green functions at their symmetry points are assumed to obey the renormalization group equations asymptotically, at least up to and including the two-loop coefficients. This is because even the two-loop $\beta$ coefficient can affect the asymptotic behavior such that an error in that coefficient would cause expressions at low energymomenta to diverge. In most cases the three- and more loop coefficients would at most give small finite corrections and can therefore be ignored at the first trial.

But we also need the elementary Green functions away from their symmetry points. In particular we must require that they do not diverge too wildly at exceptional momenta (with exceptional momenta we mean the case that the momentum-squared in one channel is much larger than in another channel, for instance $p_{12}^{2} \gg p_{13}^{2}$ ).

The two-point functions have no exceptional momenta. Suppressing Lorentz indices we require that at low momenta

$$
\left|G_{2}(p)\right| \geqq\left(p^{2}+m_{0}^{2}\right)^{2} \equiv|p|^{2}, \quad \text { if } \quad|p| \leqq \mathcal{O}\left(m_{0}\right) .
$$

At high momenta,

$$
G_{2}(p)=-|p|^{2} Z(p)^{-1}
$$

shorthand notation, to be used throughout, for momenta:

$$
\begin{gathered}
|p| \equiv \sqrt{p^{2}+m^{2}} \\
Z(p) \simeq\left(\log \left(1+\frac{|p|}{m}\right)\right)^{\sigma} \text { for large }|p| .
\end{gathered}
$$

Here $\sigma$ is the same coefficient as in (2.9) and $m$ is some finite mass which as yet we need for removing infrared divergences. (Whether or not our Feynman rules are unitary, include ghosts and the like, is irrelevant for this discussion.) Since we will arrive at one unique expression for the sum of all Feynman graphs, this sum will obey all relevant Ward-Slavnov-Taylor identities if the original Feynman rules and boundary conditions do. The sign of $\sigma$ might differ from one theory to another.

For the three- and four-point functions we have to specify precisely what happens at the exceptional momenta. There are quite a few ways in which the external momenta can be exceptional, and so we devised a compact notation. Remember that we only work in Euclidean space. Let $p_{1}, \ldots, p_{4}$ be the four 
external loop momenta. The momentum in any channel is given by the difference of two of these. The case

$$
\begin{aligned}
& \left|p_{1}-p_{2}\right| \equiv A_{1}, \\
& \left|p_{1}-p_{3}\right| \equiv A_{2}, \\
& \left|p_{1}-p_{4}\right| \equiv A_{3},
\end{aligned}
$$

with $A_{1} \ll A_{2} \ll A_{3}$, is indicated as :

$$
\left(\left((12)_{A_{1}} 3\right)_{A_{2}} 4\right)_{A_{3}} \text {, or just as }\left(\left((12)_{1} 3\right)_{2} 4\right)_{3} \text {. }
$$

If

$$
\left|p_{1}-p_{2}\right|=A_{1}, \quad\left|p_{3}-p_{4}\right|=A_{2}, \quad\left|p_{1}-p_{3}\right|=A_{3}
$$

with $A_{1} \ll A_{3} ; A_{2} \ll A_{3}$, then we write:

$$
\left((12)_{A_{1}}(34)_{A_{2}}\right)_{A_{3}} \text {, or just: } \quad\left((12)_{1}\left(34_{2}\right)_{3}\right. \text {. }
$$

And so on. The use of Schwartz' inequality for the absolute value symbol (4.3) allows us to conclude in the latter case that also

$$
\left|p_{1}-p_{4}\right| \cong\left|p_{2}-p_{3}\right| \cong\left|p_{2}-p_{4}\right| \cong A_{3} \text {. }
$$

So in our notation $A_{1,2,3}$ indicate the order of magnitude of any pair inside the labeled brackets. Table 1 now expresses the bounds that our trial functions must satisfy at exceptional momenta. The next chapter explains why they were chosen this way, and we will derive that if our trials are improved by iteration then the

Table 1. Bounds for the 3- and 4-point dressed irreducible Green functions. $Z_{i j}$ stands for $Z\left(p_{i}-p_{j}\right)$. All other exceptional momentum values can be obtained by cyclic rotations and reflections of these

$$
\begin{array}{ll}
\left((12)_{1} 3\right)_{2} & K_{1}\left(Z_{12} Z_{23} Z_{31}\right)^{-1 / 2} A_{2}\left(\frac{A_{2}}{A_{1}}\right)^{2 \eta} g\left(A_{2}\right) \\
\left(\left((12)_{1} 3\right)_{2} 4\right)_{3} & K_{2}\left(Z_{12} Z_{23} Z_{34} Z_{41}\right)^{-1 / 2}\left(\frac{A_{3}}{A_{1}}\right)^{2 \eta}\left(\frac{A_{3}}{A_{2}}\right)^{2 \zeta} g^{2}\left(A_{3}\right) \\
\left((12)_{1}(34)_{2}\right)_{3} & K_{3}\left(Z_{12} Z_{23} Z_{34} Z_{41}\right)^{-1 / 2}\left(\frac{A_{3}^{2}}{A_{1} A_{2}}\right)^{2 \eta} g^{2}\left(A_{3}\right) \\
\left(\left((13)_{1} 2\right)_{2} 4\right)_{3} & K_{4}\left(Z_{21} Z_{23} Z_{34} Z_{41}\right)^{-1 / 2}\left(\frac{A_{2} A_{3}}{A_{1}^{2}}\right)^{2 \eta+2 \zeta} g^{2}\left(A_{3}\right) \\
\left((13)_{1}(24)_{2}\right)_{3} & K_{5}\left(Z_{12} Z_{23} Z_{34} Z_{41}\right)^{-1 / 2}\left(\frac{A_{3}}{A_{1}}\right)^{4 \eta+4 \zeta} g^{2}\left(A_{3}\right) \text { if } A_{1}<A_{2} \\
\left((123)_{1} 4\right)_{2} & K_{6}\left(Z_{12} Z_{23} Z_{34} Z_{41}\right)^{-1 / 2}\left(\frac{A_{2}}{A_{1}}\right)^{2 \eta+2 \zeta} g^{2}\left(A_{2}\right) \\
\left((12)_{1} 34\right)_{2} & K_{7}\left(Z_{12} Z_{23} Z_{34} Z_{41}\right)^{-1 / 2}\left(\frac{A_{2}}{A_{1}}\right)^{2 \eta} g^{2}\left(A_{2}\right)
\end{array}
$$


new trials will again satisfy these bounds. Here $\eta$ and $\zeta$ are coefficients that must be chosen somewhere within the range

$$
\begin{aligned}
& 0<\eta<0.038, \\
& 5 \eta<\zeta<0.19 .
\end{aligned}
$$

Actually, $\zeta$ could be chosen negative, with $\zeta>-\eta$, but that would cause an unnecessary complication of our proofs. Then $g^{2}(\mu)$ is the running coupling constant which asymptotically approaches (2.8), and is assumed to be bounded by

$$
|g(\mu)| \leqq g_{0} \quad \text { for all } \mu .
$$

The numbers $K_{1}-K_{7}$ are some universal constants. The bounds for the difference functions $G_{2 \mu}, G_{2 \mu \nu}$, and $G_{3 \mu}$ are the same as for ordinary 3-and 4-point functions, except that $G_{2 \mu}$ and $G_{2 \mu \nu}$ have no factors $g$, and $G_{3 \mu}$ only one factor $g\left(A_{3}\right)$. Furthermore $Z$ factors for the newly generated external lines with the Lorentz indices are all chosen to be equal to 1 .

\section{Type III Planar Feynman Rules}

We now show that if the basic Green functions lie within the bounds given by Sect. 4, then the skeleton expansions for the 5-point functions converge. Actually we also wish to derive certain bounds for these 5-point functions at exceptional momenta. We will get these bounds as we go along. First of course we notice that the factors $Z^{ \pm 1 / 2}(p)$ in the propagators and vertices cancel. All we have to look at in this section is the powers of $A_{i}$ in Table 1 . Our argument goes much along the lines of [2]. Reference [2] would apply if $\eta=\zeta=0$. There type I and type II Feynman rules were defined. Because of the extra powers of $A_{i}$ we now define type III Feynman rules in a similar way. Because we require a proof of some extra bounds, our procedure is slightly more sophisticated than in [2]. Type III is defined as follows:

1. There are only 3 -vertices. They carry a factor 1 . Associated to them are three coefficients $\varrho_{1}, \varrho_{2}, \varrho_{3}$, belonging to the three lines. They are always in the range

$$
-\frac{1}{2}+\zeta \leqq \varrho_{i} \leqq \frac{1}{2}+3 \eta
$$

and at each vertex we require

$$
\sum \varrho_{i}=\frac{1}{2}+3 \eta
$$

2. The propagators carry a factor

$$
|k|^{-\gamma}, \quad \gamma=1-2 \eta+\varrho_{i}+\varrho_{j},
$$

where $\varrho_{i}$ and $\varrho_{j}$ are the $\varrho$ coefficients of both vertices connected by the propagator. Again we use the notation $|k|^{2}=k^{2}+m^{2}$. For practical reasons we must also require that there is a number $\mu>0$ such that

$$
\gamma \geqq \mu
$$

with in our case $\mu \leqq 4 \eta+4 \zeta$. 
3. There are no subgraphs with only two external legs. All subgraphs must be convergent by power counting. This degree of convergence, $Z$, must obey $Z \geqq \mu$, and

$$
\mu \leqq 1-6 \eta-4 \zeta
$$

The relation with the $\dot{\alpha}$ coefficients of [2] is :

$$
\varrho_{i}=\alpha_{i}+\eta \text {. }
$$

Theorem 1. The degree of convergence $Z$ of any irreducible type III diagram is given by

$$
Z=\left(\frac{3}{2}+\eta\right) E-4-\sum \varrho_{\mathrm{ext}},
$$

where $E$ is the number of external lines, and $\varrho_{\mathrm{ext}}$ are all coefficients that belong to external lines.

The proof is by trivial power counting.

Theorem 2. A planar skeleton graph with basic Green function insertions that obey the bounds of Table 1 is bounded by a type III planar graph.

This is how Table 1 was constructed. The proof goes as follows. For the 3 -point function the powers of $A_{i}$ are absorbed into the connecting propagators. We get as $\varrho$ coefficients $0,0, \frac{1}{2}+3 \eta$, or permutations thereof. The 4-point functions are rewritten as pairs of 3 -vertices connected by propagators with $\gamma \geqq 2 \zeta-2 \eta$. Now the functions of Table 1 are connected by propagators with $\gamma$ coefficients equal to 2 , but we absorb some of the powers of $A_{i}$ into these external propagators. Consequently we get external $\varrho$ coefficients ranging between

$$
\frac{1}{2}-\zeta \leqq \varrho_{\mathrm{ext}} \leqq \frac{1}{2}+3 \eta
$$

By inspection we find that all cases of Table 1 can be bounded by tree graphs with external $\varrho$ coefficients satisfying (5.8).

The internal $\varrho$ coefficients satisfy

$$
\zeta-\frac{1}{2} \leqq \varrho_{\text {int }} \leqq 3 \eta+2 \zeta-\frac{1}{2} .
$$

Now all non-trivial triangle sub-graphs that may enter from a skeleton graph must have at least two vertices that arise from 4-point elementary Green's functions and therefore have external $\varrho$ coefficients satisfying

$$
\zeta-\frac{1}{2} \leqq \varrho_{\mathrm{ext}} \leqq 3 \eta+2 \zeta-\frac{1}{2}
$$

Similarly, quadrangle graphs must have at least one original 4-vertex, and pentagons are allowed to have only 3 -vertices from the start.

The degrees of convergence derived from (5.7) are therefore bounded by

$$
\begin{aligned}
& Z_{3} \geqq 1-6 \eta-4 \zeta, \\
& Z_{4} \geqq 1-8 \eta-2 \zeta, \\
& Z_{5} \geqq 1-10 \eta .
\end{aligned}
$$


This proves that requirement No. 3 for type III diagrams is met, and so Theorem 2 is proven. Note that the coefficients $K_{i}$ must also be absorbed into the "coupling constant."

Theorem 3. There is a constant $C<\infty$, such that a diagram of type III, with $L$ loops, is bounded by $C^{L}$ times a type III tree diagram whose external $\varrho$ coefficients are either the same as those of the original diagram or $\geqq \frac{1}{2}-\zeta$. (The choice of diagram and internal $\varrho$ coefficients may depend on the external momenta.)

This is a crucial theorem for our paper. Its proof is lengthy because of bookkeeping, but goes much along the same lines as the argument of [2]. We defer the discussion of the proof to Appendix A.

\section{Finding the Elementary Green Functions at Exceptional Momenta}

In this section we regard the elementary Green functions at their symmetry points as given, and use the difference equations of Sect. 3 to express the values at exceptional momenta in terms of these. If $p_{i}-p_{j}$ is the momentum flowing through the planar channel $i j$, then in our difference equations we keep

$$
\mu=\max _{i, j}\left|p_{i}-p_{j}\right|
$$

fixed. So we express the exceptional Green functions in terms of those at the symmetry point $\mu$.

The right hand side of these difference equations again contain the elementary Green functions, also at exceptional momenta. But these only come at higher orders, and the effect of exceptional momenta is relatively small. So at this point one might already suspect that when this definition is used recursively it might converge. This will indeed be the case under certain conditions as we will show.

We wish to check whether after every next iteration the bounds of Table 1 are still obeyed. First we do this with the 4-point functions, and we consider all cases of Table 1 separately. The right hand sides of the difference equations contain 5-point functions, to which we apply Theorem 3. It says that we can replace those functions by type III tree graphs with the same external $\varrho$ coefficients. The internal structure of those graphs can still be anything as long as it obeys the type III Feynman rules. We need bounds for the absolute values of these graphs in various exceptional regions. Table 2 lists the results. The external $\varrho$ coefficients satisfy Ineq. (5.8). The power of $g^{2}\left(A_{3}\right)$ in the table anticipates that we consider the function $G_{4 \mu}$. Other functions such as $G_{3 \mu \nu}$ and $G_{2 \mu \nu \lambda}$ have one and zero powers of $g\left(A_{3}\right)$, respectively. In front of all this comes a power series of the form

$$
\sum_{n=1}^{\infty} C^{n} g_{0}^{2 n}=C g_{0}^{2}\left(1-C g_{0}^{2}\right)^{-1}
$$

which converges provided that we postulate:

$$
g_{0}=\max _{\mu}|g(\mu)|<C^{-1 / 2} .
$$


Table 2. Bounds for irreducible 5-point function at some exceptional momentum values

\begin{tabular}{ll}
\hline$\left.\left((12)_{1} 53\right)_{2} 4\right)_{3}$ & $\prod_{i} Z_{i i+1}^{-1 / 2} A_{1}^{-2 \eta} A_{3}^{2 \zeta+2 \eta} A_{2}^{-1-2 \zeta} g^{2}\left(A_{3}\right)$ \\
$\left((12)_{1} 5(34)_{2}\right)_{3}$ & $\prod_{i} Z_{i i+1}^{-1 / 2} A_{1}^{-2 \eta} A_{2}^{-2 \eta} A_{3}^{4 \eta-1} g^{2}\left(A_{3}\right)$ \\
$\left(\left((513)_{1} 2\right)_{2} 4\right)_{3}$ & $\prod_{i} Z_{i i+1}^{-1 / 2} A_{1}^{-1-4 \eta-4 \zeta} A_{2}^{2 \eta+2 \zeta} A_{3}^{2 \eta+2 \zeta} g^{2}\left(A_{3}\right)$ \\
$\left((135)_{1}(24)_{2}\right)_{3}$ & $\prod_{i} Z_{i i+1}^{-1 / 2} A_{1}^{-1-4 \eta-4 \zeta} A_{3}^{4 \eta+4 \zeta} g^{2}\left(A_{3}\right) \quad$ if $A_{2}>A_{1}$ \\
$\left((1235)_{1} 4\right)_{2}$ & $\prod_{i} Z_{i i+1}^{-1 / 2} A_{2}^{2 \zeta+2 \eta} A_{1}^{-2 \eta-2 \zeta-1} g^{2}\left(A_{2}\right)$ \\
\hline
\end{tabular}

We find, by shifting the momentum $p_{2}$ towards $p_{5}$, away from $p_{2}$ :

$$
\tilde{G}_{4}\left\{\left(\left((12)_{1} 3\right)_{2} 4\right)_{3}\right\}=G_{4}\left\{\left((523)_{2} 4\right)_{3}\right\}+\left(p_{1}-p_{5}\right)_{\mu} G_{4 \mu}\left\{\left(\left((12)_{1} 53\right)_{2} 4\right)_{3}\right\} .
$$

In this and following expressions the tilde ( $)$ indicates which quantities are being replaced by new ones in the iteration procedure.

If the Ansatz holds for $G_{4}\left\{\left((523)_{2} 4\right)_{3}\right\}$, then

$$
\begin{aligned}
\left|\tilde{G}_{4}\left\{\left(\left((12)_{1} 3\right)_{2} 4\right)_{3}\right\}\right| \leqq & K_{6}\left(Z_{45} Z_{52} Z_{23} Z_{34}\right)^{-1 / 2}\left(\frac{A_{3}}{A_{2}}\right)^{2 \eta+2 \zeta} g^{2}\left(A_{3}\right) \\
& +\left(Z_{45} Z_{12} Z_{23} Z_{34}\right)^{-1 / 2} \frac{C g_{0}^{2}}{1-C g_{0}^{2}} g^{2}\left(A_{3}\right)\left(\frac{A_{3}}{A_{1}}\right)^{2 \eta}\left(\frac{A_{3}}{A_{2}}\right)^{2 \zeta} .
\end{aligned}
$$

Choosing

$$
\frac{C g_{0}^{2}}{1-C g_{0}^{2}} \equiv \alpha,
$$

and considering that $Z_{45}=Z_{41}$, we find

$$
\begin{aligned}
\left|\tilde{G}_{4}\left\{\left(\left((12)_{1} 3\right)_{2} 4\right)_{3}\right\}\right| \leqq & \left(Z_{12} Z_{23} Z_{34} Z_{41}\right)^{-1 / 2} g^{2}\left(A_{3}\right)\left(\frac{A_{3}}{A_{1}}\right)^{2 \eta}\left(\frac{A_{3}}{A_{2}}\right)^{2 \zeta} \\
& \cdot\left(\alpha+\left(\frac{Z_{12}}{Z_{52}}\right)^{1 / 2}\left(\frac{A_{1}}{A_{2}}\right)^{2 \eta} K_{6}\right) .
\end{aligned}
$$

Let

$$
x_{12}=\left|p_{12}\right| / m \geqq 1,
$$

and

$$
f\left(x_{12}\right)=\left(\log \left(1+x_{12}\right)\right)^{\sigma / 2} \cdot x_{12}^{2 \eta} .
$$

For

$$
x_{12}>\exp (-\sigma / 4 \eta)=x_{0}
$$

this is an increasing function, so that if

$$
x_{0} m \leqq A_{1}<A_{2},
$$


then

$$
\frac{f\left(x_{12}\right)}{f\left(x_{52}\right)}<1 .
$$

The range $1 \leqq x \leqq x_{0}$ is compact, so there exists a number $L$ such that

$$
\frac{f\left(x_{12}\right)}{f\left(x_{52}\right)} \leqq L
$$

if

$$
x_{12}<x_{52} \text {. }
$$

We find

$$
K_{2} \leqq \alpha+K_{6} L
$$

Similarly we derive

$$
K_{7} \leqq \alpha+L
$$

and we use

$$
\tilde{G}_{4}\left\{\left((12)_{1}(34)_{2}\right)_{3}\right\}=G_{4}\left\{\left(52(34)_{2}\right)_{3}\right\}+\left(p_{1}-p_{5}\right)_{\mu} G_{4 \mu}\left\{\left((12)_{1} 5(34)_{2}\right)_{3}\right\}
$$

to derive

$$
K_{3} \leqq \alpha+K_{7} L \leqq \alpha+\alpha L+L^{2}
$$

and for the three point function,

$$
K_{1} \leqq K_{7}+L \leqq \alpha+2 L
$$

The remaining coefficients $K_{4-6}$ must be computed in a slightly different way. Consider $K_{4}$. We replace $p_{1}$ by $p_{5}$ now in such a way that

$$
\left|p_{5}-p_{3}\right| \simeq 2\left|p_{1}-p_{3}\right| ; \quad A_{1} \rightarrow 2 A_{1},
$$

and work with induction. Then

$$
\tilde{G}_{4}\left\{\left(\left((13)_{1} 2\right)_{2} 4\right)_{3}\right\}=\tilde{G}_{4}\left\{\left(\left((53)_{1} 2\right)_{2} 4\right)_{3}\right\}+\left(p_{1}-p_{5}\right)_{\mu} G_{4 \mu}\left\{\left(\left((513)_{1} 2\right)_{2} 4\right)_{3}\right\},
$$

and we find, again inspecting Table 2 ,

$$
K_{4}\left(1-2^{-4 \eta-4 \zeta}\right)=\alpha
$$

[actually there might be an unimportant extra factor close to one here, and (6.22) holds unless the bound given by $K_{4}$ would become smaller than the one given by $K_{6}$.

Applying the same technique we compute a bound for $K_{5}$, but we must separate in turn $p_{1}$ from $p_{3}$ and $p_{2}$ from $p_{4}$. This makes the rate of convergence slower:

$$
K_{5}\left(1-2^{-4 \eta-4 \zeta}\right)=2 \alpha
$$


Finally $K_{6}$ is achieved by widening the separation between $p_{1}, p_{2}$, and $p_{3}$, in successive steps of factors of 2 :

$$
\begin{aligned}
\tilde{G}_{4}\left\{\left((123)_{1} 4\right)_{2}\right\}= & \tilde{G}_{4}\left\{\left((563)_{1} 4\right)_{2}\right\} \\
& +\left(p_{5}-p_{1}\right)_{\mu} G_{4 \mu}\left\{\left((1235)_{1} 4\right)_{2}\right\} \\
& +\left(p_{6}-p_{2}\right)_{\mu} G_{4 \mu}\left\{\left((2356)_{1} 4\right)_{2}\right\},
\end{aligned}
$$

(where $\left|p_{5}-p_{6}\right|=2\left|p_{1}-p_{2}\right| ;\left|p_{6}-p_{3}\right|=2\left|p_{2}-p_{3}\right| ;\left|p_{3}-p_{5}\right|=2\left|p_{3}-p_{1}\right|$ )

$$
\begin{aligned}
& \left|\tilde{G}_{4}\left\{\left((123)_{1} 4\right)_{2}\right\}\right| \leqq \alpha Z_{1}^{-1} Z_{2}^{-1}\left(\frac{A_{2}}{A_{1}}\right)^{2 \eta+2 \zeta} \\
& \sum_{n=1}^{{ }^{2} \log \left(A_{2} / A_{1}\right)}\left(2^{-(2 \eta+2 \zeta) n} \cdot 2 \frac{Z\left(A_{1}\right)}{Z\left(2^{n} A_{1}\right)}\right)+\left|G_{4}\left\{(1234)_{2}\right\}\right| .
\end{aligned}
$$

The sum certainly can be bounded:

$$
\sum \leqq L^{\prime} \leqq 2 L\left(1-2^{-2 \zeta}\right)^{-1}
$$

Therefore:

$$
K_{6} \leqq \max \left(1, \alpha L^{\prime}\right) .
$$

Thus all coefficients $K_{i}$ have been determined. Note that these coefficients may blow up if $\eta, \zeta \rightarrow 0$, and this would have to be compensated by choosing $g_{0}$ small enough. So at finite but small enough $g_{0}$ we find some non-vanishing coefficients $\eta, \zeta, K_{i}$ that bound our amplitudes, and only if $g^{2} \rightarrow 0$ then $\eta, \zeta \rightarrow 0$. It will be clear from the above arguments that our bounds are only very crude. Our present aim was only to establish their existence and not to find optimal bounds.

What has been shown in this section is that if our first trials for the elementary Green functions obey the bounds of Table 1, and if Eqs. (6.4), (6.17), (6.21), and (6.24) are used to find other trial functions, then these again obey the bounds.

In Sect. 8 we show that the series of trial Green functions thus obtained converges geometrically to a certain limit.

\section{Non-Exceptional Momenta}

In order to formulate the complete recursion procedure for determining the basic Green functions we need relations that link these Green functions at different symmetry points. Again the difference equations are used:

$$
\begin{aligned}
G_{4}\left(p_{1} \ldots p_{4}\right) & =G_{4}\left(2 p_{1}, p_{2} p_{3} p_{4}\right)-p_{\lambda} G_{4 \lambda}\left(p_{1}, 2 p_{1}, p_{2} p_{3} p_{4}\right) \\
& =\ldots=G_{4}\left(2 p_{1}, \ldots, 2 p_{4}\right)-\sum_{i=1}^{4} p_{i \lambda} G_{4 \lambda}\left(p_{1}^{(i)}, \ldots, p_{5}^{(i)}\right) .
\end{aligned}
$$

Here $p_{i}$ and $p_{j}^{(i)}$ are external loop momenta. They are non-exceptional. We use a shorthand notation for (7.1), writing $p_{i}^{2} \simeq\left(p_{i}-p_{j}\right)^{2} \simeq \mu^{2}$ :

$$
G_{4}(\mu)-G_{4}(2 \mu)=-\mu \sum_{i=1}^{4} G_{4 \lambda}^{(i)}(2 \mu, \mu) .
$$


Similarly we have

$$
G_{2,3}(\mu)-G_{2,3}(2 \mu)=-\mu \sum_{i} G_{2,3 \lambda}^{(i)}(2 \mu, \mu) .
$$

These are just discrete versions of the renormalization group equations. The right hand side of (7.2), [not (7.3)!] is to be expanded in a skeleton expansion which contains all basic Green functions at all $\mu$, also away from their symmetry points. There we insert the values obtained after a previous iteration. We wish to show now that all diagrams that make up the right hand side of (7.2) decrease sufficiently rapidly as $\mu \rightarrow \infty$. Since $G_{4}$ at the symmetry point is defined by our running coupling constant(s), Eq. (7.2) can be expected to yield an equation of the form

$$
\frac{\mu \partial}{\partial \mu} g_{i}(\mu)=-\sum_{l=2}^{k} \beta_{i j_{1} \ldots j_{l}}^{(l)} g_{j_{1}}(\mu) \ldots g_{j_{l}}(\mu)+|g(\mu)|^{N} \varrho_{i}(\mu),
$$

where $\beta^{(l)}$ are the first $k$ coefficients, which coincide with the perturbatively computed $\beta$ coefficients [with an apology: the definition of $l$ is not the same as in Eqs. (2.9) and (2.11)]. Usually only odd powers occur, so that $k=N-2$. The rest function $\varrho$ satisfies

$$
|\varrho(\mu)| \leqq Q_{N},
$$

for some constant $Q_{N}<\infty$. This inequality must hold in the sense that $|g(\mu)|^{N} \varrho(\mu)$ must be a convergent expansion in the functions $g\left(\mu^{\prime}\right)$, with

$$
\mu^{\prime} \geqq m
$$

(so that $\mu^{\prime}$ may be smaller than $\mu$ ), in such a way that the absolute value of each diagram contributes to $Q_{N}$ and their total sum remains finite.

Now clearly Eq. (7.2) is a difference equation, not a differential equation such as (7.4). Up till now differential equations were avoided because of infrared divergences. Just for ease of notation we have put (7.4) in differential form because the mathematical convergence questions that we are to consider now are insensitive to this simplification.

Consider the skeleton expansion of $G_{4 \lambda}^{(i)}$ in (7.2). At each of the four external particle lines a factor $g\left(\mu_{i}\right)$ occurs with $\mu_{i} \geqq \mu$, so it may seem easy to prove (7.4) from (7.2) with $N=3$ or 4 . However, we find it more convenient ${ }^{3}$ to have an equation of the form (7.4) with $N \leqq 7$, and our problem is that the internal vertices of the $G_{4 \lambda}^{(i)}$ might have momenta which are all less than $\mu$. We will return to this question.

In proving the difference equation variant of (7.4) from (7.2) we have to make the transition from $G_{4}$ to $g^{2}$ and $G_{3}$ to $g$, and this involves the coefficients $Z(\mu)$, associated to the functions $G_{2}$, by equations of the form

$$
\begin{aligned}
& G_{2}(\mu)=-\mu^{2} Z^{-1}(\mu) ; \\
& G_{3}(\mu)=\mu Z^{-3 / 2}(\mu) g_{3}(\mu) ; \\
& G_{4}(\mu)=Z^{-2}(\mu) g_{4}^{2}(\mu),
\end{aligned}
$$

3 Closer analysis shows that actually $N=3$ or 4 is sufficient to prove unique solubility. Only if we wish an exact, non-perturbative definition of the free parameters we need the higher $N$ values. Note that not only $Q_{N}$ but also $g_{0}$ may deteriorate as $N$ increases 
where $g_{3}, g_{4}$ are just various components of the coupling constant $g_{i}$. In the following expressions we suppress these indices $i$ when we are primarily interested in the dependence on $\mu(=|p|$ at the symmetry point). Now from (7.2) and (7.3) we find not first order but third order differential equations for $G_{2}$, basically of the form

$$
\frac{\partial^{3}}{\partial \mu^{3}} G_{2}=-G_{2, \lambda \lambda \lambda}=\mathcal{O}\left(g^{2}(\mu) Z^{-1}(\mu) / \mu\right)
$$

where $G_{2, \lambda \lambda \lambda}$ is just a shorthand notation for the combination of expandable functions $G_{2, \lambda \mu v}$ obtained after taking differences three times. Write

$$
U_{2}(\mu)=-\frac{\partial^{2}}{\partial \mu^{2}} G_{2}(\mu)=-G_{2 \lambda \lambda}(\mu),
$$

then

and

$$
\frac{\mu \partial}{\partial \mu} U_{2}(\mu)=-\mu G_{2, \lambda \lambda \lambda}(\mu)
$$

$$
\mu^{2} Z^{-1}(\mu)=\int_{m}^{\mu}\left(\mu-\mu_{1}\right) U_{2}\left(\mu_{1}\right) d \mu_{1}+A \mu+B .
$$

Here $A$ and $B$ are free integration constants; $A$ is usually determined by Lorentz invariance and $B$ by the mass, fixed to be equal to $m$. In lowest order:

$$
A=m U_{2}(m) ; \quad B=-\frac{1}{2} m^{2} U_{2}(m) .
$$

This strange-looking form of the integration constants is an artifact coming from our substitution of difference equations by differential equations. Using difference equations we can impose Lorentz invariance by symmetrization in momentum space, so that only one (for each particle) integration constant is left: the mass term. We choose at all stages $\frac{1}{2} U_{2}(m)=Z(m)=1$.

A convenient way to implement Eq. (7.12) is to formally define $U_{2}(\mu)=2$ if $0 \leqq \mu \leqq m$, and replace the lower bound of the integral in (7.11) by zero. Then after symmetrization: $A=B=0$.

Equation (7.11) has a linearly convergent integral, whereas (7.10) is logarithmic. Together they determine the next iterative approximation to $G_{2}$. In fact we have

$$
\mu G_{2, \lambda \lambda \lambda}(\mu)=Z^{-1}(\mu) f(\{g\}),
$$

and in $f(\{g\}), Z$ occurs only indirectly. So the iteration converges fastest if we replace $(7.10)$ by

$$
\frac{\mu \partial}{\partial \mu} \tilde{U}_{2}(\mu)=-\mu \frac{Z(\mu)}{\tilde{Z}(\mu)} G_{2, \lambda \lambda \lambda}(\mu),
$$

where the tilde denotes the new function $U_{2}(\mu)$.

One can however also use (7.9) with $U_{2}$ replaced by $\tilde{U}_{2}$.

We find

$$
\frac{\mu \partial}{\partial \mu} Z^{-1}=-\int_{m / \mu}^{1} d \tau(1-\tau) \mu G_{2, \lambda \lambda \lambda}(\tau \mu) .
$$

As stated before, the $\mathcal{O}\left(\frac{m}{\mu}\right)$ terms have been removed by symmetrization. 
This equation allows us to remove the $Z$ factors from the functions $G_{3,4}$ and arrive at first order renormalization group integrodifferential equations for $g_{i}(\mu)$.

For the 3-point functions we must write

$$
\begin{gathered}
U_{3}(\mu)=G_{3, \lambda}(\mu)=\frac{\partial G_{3}}{\partial \mu}, \\
\frac{\mu \partial}{\partial \mu} U_{3}(\mu)=\mu G_{3, \lambda \lambda}(\mu), \\
G_{3}(\mu)=\int_{m}^{\mu} \mu U_{3}(\mu) d \mu+C_{3}, \\
\frac{\mu \partial G_{3}(\mu)}{\partial \mu}=\int_{m / \mu}^{1} d \tau \mu G_{3, \lambda \lambda}(\tau \mu) .
\end{gathered}
$$

A potential difficulty in writing down the renormalization group equation even for $N=4$ is the convolutions in (7.15) and (7.18) which contain Green functions at lower $\mu$ values, and so they depend on $g\left(\mu^{\prime}\right)$ with $\mu^{\prime}<\mu$. So a further trick is needed to derive (7.4). This is accomplished by realizing that the integrals in (7.15) and (7.18) converge linearly in $\mu$. Suppose we require at every iteration step

$$
\left|\frac{\mu \partial}{\partial \mu} g(\mu)\right| \leqq \hat{\beta}|g(\mu)|^{3} \quad \text { and } \quad|g(\mu)| \leqq g_{0}
$$

for some $\hat{\beta}<\infty, g_{0}<\infty$. Then it is easy to show that if $\mu_{1} \leqq \mu$, then

$$
\left|g\left(\mu_{1}\right)\right| \leqq|g(\mu)|+C\left(\frac{\mu}{\mu_{1}}\right)^{\varepsilon}\left|g^{3}(\mu)\right|,
$$

if

$$
\varepsilon \geqq 3 \hat{\beta} g_{0}^{2}+\hat{\beta} / C \text {. }
$$

So with $C$ large enough and $g_{0}$ small enough we can make $\varepsilon$ as small as we like. Inequality (7.20) is proven by differentiating with $\mu$. This enables us to replace $g(\tau \mu)$ by $g(\mu)$ in (7.15) and (7.18) while the factor $\tau^{-\varepsilon}$ does no harm to our integrals. So we find bounds for $\frac{\mu \partial}{\partial \mu} \tilde{Z}^{-1}$ and $\frac{\mu \partial}{\partial \mu} G_{3}$ in terms of a power series of $g(\mu)$. We must terminate the series as soon as the factors $\tau^{-\varepsilon}$ accumulate to give $\tau^{-1}$. This implies that $N$ must be kept finite, otherwise $g_{0} \rightarrow 0$.

The same inequality (7.16) is used to go from $N=4$ to $N=7$ in these equations. If in a skeleton diagram a vertex is not associated with any external line, then it may be proportional to a factor $g\left(\mu^{\prime}\right)$ with $\mu^{\prime}<\mu$. But using (7.16) we see that it may be replaced by $g(\mu)$ at the cost of a factor $\left(\mu / \mu_{1}\right)^{\varepsilon}$. At most three of these extra factors are needed. If the three corresponding vertices are chosen not to be too far away from one of the external vertices of the diagram (which we can always arrange), then this just corresponds to inserting an extra factor $\left(\frac{p^{\text {ext }}}{p_{1}}\right)^{\varepsilon}$ at an external vertex. This in turn means a replacement $\eta \rightarrow \eta+\frac{1}{2} \varepsilon$, so if $\eta$ was not chosen 
to be maximal, then Theorem 3 remains valid and the skeleton expansion still converges.

From the above considerations we conclude that an equation of the form (7.4) can be written down for any finite $N$, such that $Q_{N}$ in inequality (7.5) remains finite. We do expect of course that $Q_{N}$ might increase rapidly with $N$, but then we only want the equation for $N \leqq 7$. We are now in a position to formulate completely our recursive definition of the Green functions $G_{2}, G_{3}, G_{4}$ of the theory:

1) We start with a given set of trial functions $G_{2}(\mu), G_{3}(\mu), G_{4}(\mu)$ for the basic Green functions at their symmetry points. These must satisfy the boundary conditions of Sect. 2 at $\mu \rightarrow \infty$, and Eq. (7.19). We use the procedure of Sect. 6 to determine $G_{2,3,4}$ at the exceptional momenta, that is, we start with some trial function at the exceptional momenta as well and apply the procedure of Sect. 6 recursively, to obtain better and better values. The convergence of that will be shown in Sect. 8a.

2) Knowing how to compute the exceptional Green functions from $G_{2,3,4}$, we are now able to find the right hand side of the renormalization group equation for $G_{2}$, or rather $Z^{-1}$, from (7.15), using (7.20):

$$
\frac{\mu \partial}{\partial \mu} \tilde{Z}_{(i)}^{-1}(\mu)=\tilde{Z}_{(i)}^{-1}(\mu)\left(\gamma_{i j k} g_{j}(\mu) g_{k}(\mu)+g^{4}(\mu) \sum(\mu)\right),
$$

where $\sum(\mu)$ is again bounded. Here $\gamma_{i j k}$ are the one-loop gamma coefficients. This gives us the $Z$ functions if $g_{i}(\mu)$ are known. In Sect. $8 \mathrm{~b}$ we briefly discuss existence of solutions to (7.22).

3) This then enables us to compute the right hand side of Eq. (7.4). Before integrating (7.4) it is advisable to apply Ward identities (if we were dealing with a gauge theory) in order to reduce the number of independent degrees of freedom at each $\mu$. As is well known, in gauge theories one can determine all subtraction constants this way except those corresponding to the usual free coupling constants and gauge fixing parameters [10]. So the number of unknown functions $g_{i}(\mu)$ need not exceed the number of "independent" dimensionless coupling constants of the theory ${ }^{4}$.

4) We now solve Eq. (7.4), by an iterative procedure, of which Sect. 8c discusses the details.

We must find out under what conditions this recursive procedure converges.

\section{Convergence of the Procedure}

\section{a) Exceptional Momenta}

In Sect. 6 a procedure is outlined to obtain the Green functions at exceptional momenta, if the Green functions at the symmetry points are given. That procedure is recursive because Eqs. (6.4), (6.17), (6.21), and (6.24) determine the Green functions $G_{2,3,4}$ in terms of the symmetry ones, and $G_{4 \mu}, G_{3 \mu \nu}, G_{2 \mu \nu \lambda}$. But the latter still contain the previous ansatz for $G_{2,3,4}$. Fortunately it is easy to show that any error $\delta G_{2,3,4}$ will reduce in size, so that here the recursive procedure converges.

4 We put "independent" between quotation marks because our requirement of asymptotic freedom usually does give relations among various running coupling constants 
Let us indicate the bounds discussed in Sects. 4 and 6 as

$$
\left|G_{n}\left(p_{1}, \ldots, p_{n}\right)\right| \leqq B_{n}\left(p_{1}, \ldots, p_{n}\right),
$$

and assume that a first trial $G_{n}^{(1)}$ has an error

with some $\varepsilon^{(1)} \leqq 1$.

$$
\left|\delta G_{n}^{(1)}\right| \leqq \varepsilon^{(1)} B_{n}
$$

Now $G_{4 \mu}, G_{3 \mu \nu}, G_{2 \mu \nu \lambda}$ also satisfy inequalities of the form (8.1). Furthermore they were one order higher in $g^{2}$. So we have

$$
\left|\delta G_{4 \mu}\right| \leqq \varepsilon^{(1)} C g^{2}(\mu) B_{4 \mu},
$$

where $C$ is linked to the universal convergence coefficient. We indicated a factor $g^{2}(\mu)$ explicitly, implying that $B_{4 \mu}$ may be independent of $g^{2}(\mu)$, and with $\left|G_{4 \mu}\right| \leqq B_{4 \mu}$ one could prove the bound (8.1), as shown in Sect. 6. So the new error will be bounded by

$$
\left|\delta G_{n}^{(2)}\right| \leqq \varepsilon^{(1)} C g^{2}(\mu) B_{n}
$$

So as soon as

$$
\left|C g_{0}^{2}\right|<1,
$$

where $g_{0}$ is the upper bound for $g(\mu)$, our procedure converges. We stress that the above argument is only valid as long as the Green functions at the symmetry points were kept fixed, and are determined by $g(\mu)$, with $\mu=\max _{\text {channels }}\left|p_{i j}\right|$.

\section{b) The Z Factors}

Knowing that at any stage $g(\mu)$ satisfies Ineq. (7.19), we find that the solution of $(7.22)$ is

$$
\begin{aligned}
\log \tilde{Z}_{i}(\mu) & =\int^{\mu} d \log \mu_{1}\left(\gamma_{i j k} g_{j}\left(\mu_{1}\right) g_{k}\left(\mu_{1}\right)+g^{4}\left(\mu_{1}\right) \sum\left(\mu_{1}\right)\right) ; \\
\tilde{Z}_{i}(\mu) & =\left(\log \frac{\mu}{\Lambda}\right)^{\sigma_{l}}\left(1+\mathcal{O}\left(g^{2}\right)\right),
\end{aligned}
$$

where the $\mathcal{O}\left(g^{2}\right)$ terms are again bounded by a coefficient times $g^{2}(\mu)$. These equations must be solved iteratively, because the right hand side of (8.6) contains skeleton expansions that again contain $Z(\mu)$, hidden in the function $\sum\left(\mu_{1}\right)$. It is not hard to convince oneself that such iterations converge. A change

$$
|\delta Z| \leqq \varepsilon^{(1)} g^{2} Z
$$

yields a change in the function $\sum\left(\mu_{1}\right)$ bounded by

$$
\left|\delta \sum\right| \leqq \varepsilon^{(1)} g_{0}^{2} \sum \text {, }
$$

so that

$$
\frac{\delta \tilde{Z}(\mu)}{\tilde{Z}(\mu)} \leqq C \varepsilon^{(1)} g_{0}^{2} g^{2}(\mu) \leqq \varepsilon^{(2)} g^{2}(\mu)
$$

with $\varepsilon^{(2)}<\varepsilon^{(1)}$ if $g_{0}$ is small enough. 
c) $g_{i}(\mu)$

We now consider the integro-differential equation (7.4). The solution is constructed by iteratively solving

$$
\frac{\mu \partial}{\partial \mu} \tilde{g}_{i}(\mu)+\sum_{l=2}^{k} \beta_{i j_{1} \ldots j_{l}}^{(l)} \tilde{g}_{j_{1}}(\mu) \ldots \tilde{g}_{j_{l}}(\mu)=|g(\mu)|^{N} \varrho_{i}(\mu),
$$

where the tilde denotes the next "improved" function $g_{i}(\mu)$. Our first ansatz will be a solution of (8.11) with $\varrho\{g\}$ replaced by zero. This certainly exists because the $\beta$-coefficients, of which we only need the first two, are determined by perturbation expansion and therefore finite. The integration constant must be chosen such that for all $\mu \geqq m$ we have

$$
\left|g^{2}(\mu)\right| \leqq \kappa g_{0}^{2} ; \kappa<1,
$$

with some boundary condition at $\mu=\infty$ as given by Eqs. (2.10)-(2.14). (It is instructive to consider also complex $\Lambda$.)

If we now substitute this $g(\mu)$ into the right hand side of (8.11) we find a correction

$$
g(\mu) \rightarrow \tilde{g}(\mu)=g(\mu)+\delta g(\mu) .
$$

We can require the boundary condition

$$
|\delta g(\mu)| \leqq \varepsilon A|g(\mu)|^{N-2}, \text { for all } \mu,
$$

such that

$$
A|g(\mu)|^{N-2} \leqq|g(\mu)|, \quad \text { for all } \mu .
$$

Under what conditions does a recursive application of (8.11) give a stable solution? Let the first ansatz produce a change (8.14). The next correction is then, up to higher order in $\delta g$, given by

$$
\frac{\mu \partial}{\partial \mu} \delta \tilde{g}_{i}(\mu)+M_{i j}(\mu) \delta \tilde{g}_{j}(\mu)=\delta f_{i}(\mu),
$$

where

$$
M_{i j}(\mu)=\sum_{l} l \beta_{i j k}^{(l)} \tilde{g}_{k}(\mu) \ldots
$$

To estimate $\delta f(\mu)$ we must find a limit for the change in $\varrho$. Our argument that

$$
|\varrho|<C
$$

came from adding the absolute values of all diagrams contributing to $\varrho$, so therefore we can be sure that, from (8.14) and (8.15),

$$
|\delta \varrho|<\varepsilon C^{\prime},
$$

with $C^{\prime}$ slightly larger than $C$, and

$$
|\delta f(\mu)| \leqq \varepsilon(N+1) C^{\prime} g(\mu)^{N} .
$$

Now asymptotically,

$$
M_{i j}(\mu) \rightarrow M_{i j}^{0} / \log \mu,
$$


where $M_{i j}^{0}$ is determined by one-loop perturbation theory. If there is only one coupling constant it is the number $3 / 2$. In the more general case we now assume it to be diagonalized:

$$
M_{i j}^{0}=M(i) \delta_{i j},
$$

with one eigenvalue equal to $3 / 2$. (Our arguments can easily be extended to the special situation when $M_{i j}^{0}$ cannot be diagonalized, in which case the standard triangle form must be used.) The asymptotic form of the solution to (8.16) is

$$
\delta \tilde{g}_{i}(\mu)=(\log \mu)^{-M(i)} \int_{\mu(i)}^{\mu} d \log \mu(\log \mu)^{M(i)} \delta f_{i}(\mu),
$$

where $\mu(i)$ are integration constants. If $M(i)<\frac{N}{2}-1$ then we choose $\mu(i)=\infty$. If $M(i)>\frac{N}{2}-1$ we set $\mu(i)=m$. Then in both cases we get

$$
\left|\delta \tilde{g}_{i}(\mu)\right|<\frac{\varepsilon(N+1) C^{\prime \prime}}{\left|\frac{N}{2}-M(i)-1\right|}|g(\mu)|^{N-2},
$$

where $C^{\prime \prime}$ is related to $C^{\prime}$ and the first $\beta$ coefficient. In a compact set of $\mu$ values where the deviation from (8.23) is appreciable we of course also have an inequality of the form (8.24).

If $M(i)=N / 2-1$, then our first ansatz must be chosen slightly better than (8.14) replacing $N$ by a larger value.

Comparing (8.24) with (8.14), noting that $C^{\prime \prime}$ is independent of $A$, we see that if

$$
A>\frac{(N+1) C^{\prime \prime}}{\left|\frac{N}{2}-M(i)-1\right|},
$$

then our procedure converges. $A$ is determined by imposing the bound (8.15). It can be chosen large by choosing $g_{0}$ small:

$$
A=g_{0}^{3-N} \text {. }
$$

So $N>3$, and Eq. (8.14) can only be true if $N>5$. Usually, $N$ and $l$ in (7.4) are odd, so $N \geqq 7$.

We close this section with a mere formality: Ineq. (7.19) has to be checked at every iteration for $g(\mu)$. But it holds almost by construction, since our improved versions $\tilde{g}(\mu)$ satisfy (8.11).

\section{Discussion}

What has been proven is that there are non-trivial values for the masses and coupling constants for which a recursive procedure exists that yields solutions to the imposed difference equations for the Green functions. All skeleton expansions that are needed to produce this solution converge, and all convergence rates are 
geometric. The finite mass values were only needed in order to limit the growth of $g(\mu)$ at small $\mu$ values, such that

$$
|g(\mu)| \leqq g_{0}
$$

everywhere. However, (9.1) can also be achieved by using complex solutions rather than real ones because then the singularity in $g(\mu)$ moves to a harmless place in the complex plane. In that case then the mass is not necessary and this enabled us in [4] to draw some conclusions on the Borel functions for massless planar QCD. However a complete convergent formalism for massless planar QCD is still lacking.

Our convergence proofs were performed by deriving bounds on convergence rates, but these bounds are still extremely crude (roughly at every estimate in our paper $g_{0}$ had to decrease by a factor of 2). We made no serious attempts to find the tightest possible bounds or to actually estimate the true convergence rate.

\section{Appendix A. Proof of Theorem 3}

The proof goes by induction. We successively replace loops by trees. We begin by removing all triangular loops, replacing them by single 3-vertices, until the only subgraphs with three legs contain more than one facet. If such a subgraph appears we replace it by a single triangle by applying the entire process described here to that subgraph first.

The replacement of a triangle loop by a vertex goes as follows. Equations (5.4) and (5.5) can be written as

$$
\begin{aligned}
& Z \geqq \mu ; \\
& \gamma \geqq \mu ; \\
& \mu=\min (2 \zeta-2 \eta, 1-6 \eta-4 \zeta) .
\end{aligned}
$$

The most divergent subgraphs adjacent to our triangle are labled 1,2,3 (see Fig. A1).

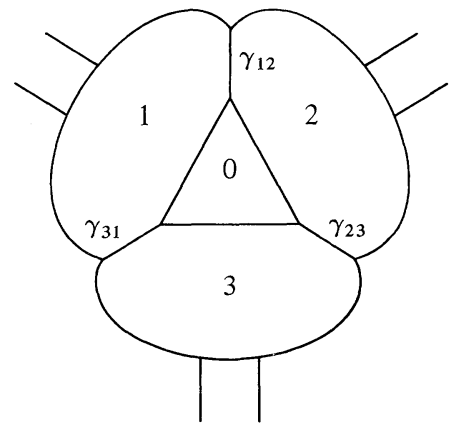

a

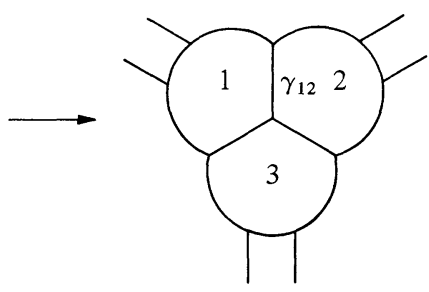

b

Fig. A $1 \mathbf{a}$ and b. Triangle loop with its most divergent surrounding subgraphs $\mathbf{a}$. Here $\gamma_{i j}$ are the powers associated with the propagators shared by subgraphs $i$ and $j$. It is bounded by the vertex at $\mathbf{b}$, where the new propagators have powers $\bar{\gamma}_{i j}=\gamma_{t j}+\delta_{k}(i, j, k$ cyclic $)$ 

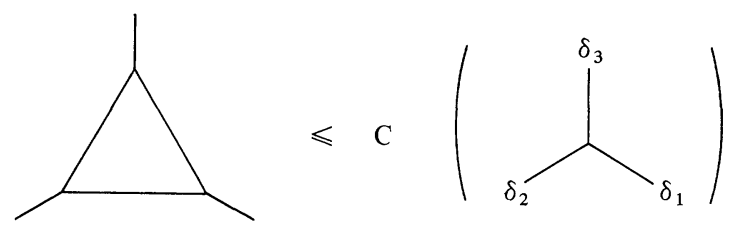

Fig. A2. Triangles with $Z<4$ are bounded by vertices with $\sum \delta_{l}=Z$

Let $Z_{i}$ be the degree of convergence of subgraph $i$;

$Z_{0 i}$ be the degree of convergence in the combined graph $(i)+(0)$. Let $Z_{\text {tot }}$ be the degree of convergence of the graph made up by $0,1,2,3$ together.

Then power counting gives us

$$
\sum_{i} Z_{0 i}=Z_{\text {tot }}+2 Z_{0}+\sum \gamma_{i i+1} \geqq 3 \mu+Z_{0} .
$$

Here $\gamma_{12}$ is the power attached to the propagator shared by 1 and 2, etc. The inequality holds because all $Z$ and $\gamma$ satisfy (A.1). Now the triangle is replaced according to Fig. A2, the powers $\delta_{i} \geqq 0$ must satisfy [2]

$$
\sum \delta_{i}=Z_{0} \text {. }
$$

We choose them such that $Z_{0 i} \geqq \mu+\delta_{i}$, so that the new divergences in diagram b) still satisfy $\bar{Z}_{i} \geqq \mu$. (Here the bar refers to the diagram of Fig. A 1b.)

Now the inequality of Fig. A 2 only holds [2] if also

$$
\delta_{2}+\delta_{1}<\gamma_{03}
$$

and permutations thereof. This can only be violated if

$$
\gamma_{01}+\gamma_{02} \geqq 4
$$

because only then

$$
Z \geqq \gamma_{03} \text {. }
$$

But

$$
\begin{aligned}
\gamma_{01}+\gamma_{02} & \leqq 2\left(1-2 \eta+\frac{1}{2}+3 \eta\right)+\frac{1}{2}+3 \eta-\varrho_{\min } \\
\varrho & \geqq-\frac{1}{2}+\zeta ; \\
\gamma_{01}+\gamma_{02} & \leqq 4+5 \eta-2 \zeta .
\end{aligned}
$$

So if $\zeta>5 \eta$ according to (4.9) there is no problem. After removal of triangles this way Ineqs. (A.1) still hold.

Now before removing the quadrangle we first remove all negative $\varrho$ coefficients except those at the external vertices of our diagram (or our triangular subdiagram), using the inequality of Fig. A3, where $\varrho_{1}<0$. This is simply the inequality

$$
\left|p_{2}+p_{3}\right|^{-\varrho_{1}} \leqq p_{2}^{-\varrho_{1}}+p_{3}^{-\varrho_{1}} \text {. }
$$




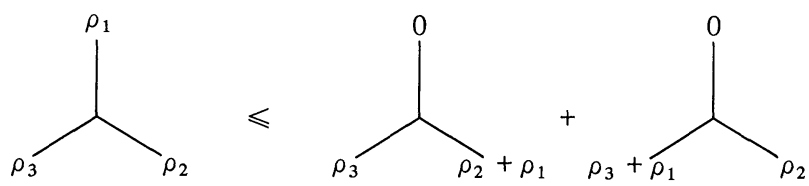

Fig. A3. Inequality (A10). The coefficients $\varrho_{i}$ are indicated
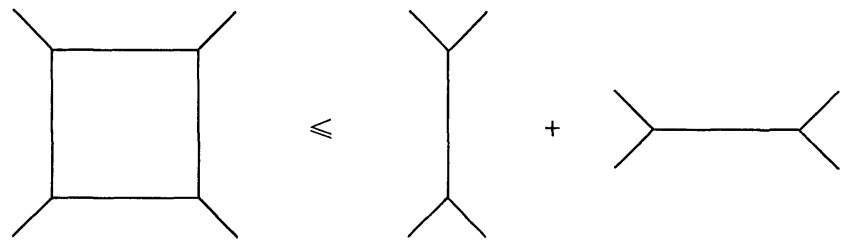

Fig. A4. Inequality for quadrangle loop with $Z<4$

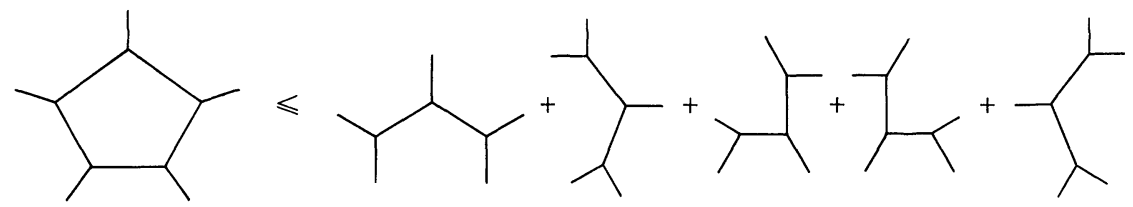

Fig. A5. Inequality for pentagons
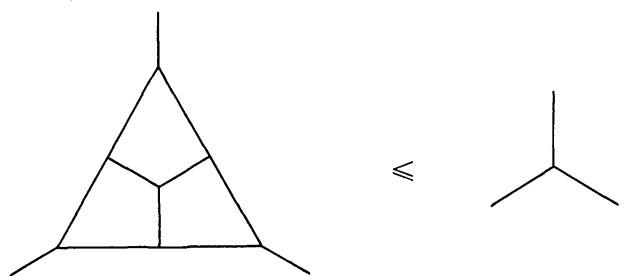

Fig. A6. Special case: three quadrangles in a triangle

The new $\varrho$ coefficients at the right hand side are all non-negative. Inequality (A.1) for quadrangular subgraphs remains valid. Replacement of quadrangular subloops now goes exactly as in [2] (see Fig. A4). One is entirely free to choose the new external $\varrho$ coefficients in the tree graphs either equal to or larger than those of the loop. As in [2] chains of quadrangular graphs ("generalized ladder graphs") are removed inductively. Also removal of pentagons is identical to the procedure of [2]. The proof given there applies without important changes (Fig. A 5).

One complication arises because we might have negative $\varrho$ coefficients at the boundary of the graph. These are simply kept unchanged. It is easy to verify that quadrangles and pentagons that have propagators in common with the edge of our graph can be handled without complications. We needed $\varrho \geqq 0$ only at least two corners of the quadrangular graphs because then $Z<4$, so that Fig. A 4 holds. All $\varrho$ coefficients are always kept $\geqq-\frac{1}{2}+\zeta$.

When these procedures are applied to a non-trivial triangular subgraph, one might end up with a configuration pictured in Fig. A6, where three quadrangles 
combine to from a triangle. This we had not yet discussed, but bounding it by a vertex using the loop-tree inequalities of [2] is straightforward.

Acknowledgement. The author thanks P. van Baal for discussions and criticism.

\section{References}

1. 't Hooft, G. : Is asymptotic freedom enough? Phys. Lett. 109B, 474 (1982)

2. 't Hooft, G.: On the convergence of planar diagram expansions. Commun. Math. Phys. 86, 449 (1982)

3. Eguchi, T., Kawai, H.: Phys. Rev. Lett. 48, 1063 (1982)

Parisi, G. : A simple expression for planar field theories. Phys. Lett. 112B, 463 (1982)

4. 't Hooft, G.: Borel summability of a four-dimensional field theory. Utrecht preprint, September 1982, Phys. Lett. B (to be published)

5. de Calan, C., Rivasseau, V.: Local existence of the Borel transform in Euclidean $\phi_{4}^{4}$. Commun. Math. Phys. 82, 69 (1981)

6. For definitions of these properties, see Symanzik, K.: Renormalizable models with simple symmetry breaking. I. Symmetry breaking by a source term. Commun. Math. Phys. 16, 48 (1970) Symanzik, K. : In: Cargèse Lectures in Physics, Vol. 6. Bessis, J.D. (ed.). New York: Gordon and Breach 1971

7. 't Hooft, G. : A planar diagram theory for strong interactions. Nucl. Phys. B72, 461 (1974)

8. Gell-Mann, M., Low, F.: Quantum electrodynamics at small distances. Phys. Rev. 95, 1300 (1954)

9. Politzer, H.D.: Asymptotic freedom: An approach to strong interactions. Phys. Rep. 14C, 129 (1974)

Narison, S.: Techniques of dimensional regularization and the two-point functions of QCD and QED. Phys. Rep. 84, 263 (1982)

Jones, D.R.T.: Two-loop diagrams in Yang-Mills theory. Nucl. Phys. B75, 531 (1974)

Caswell, W.E.: Asymptotic behavior of non-abelian gauge theories to two-loop order. Phys. Rev. Lett. 33, 244 (1974)

van Damme, R. : Most general two-loop counterterm for Fermion-free gauge theories with scalar fields. Phys. Lett. 110B, 239 (1982)

10. 't Hooft, G. : Renormalization of massless Yang-Mills fields. Nucl. Phys. B33, 173 (1971)

Communicated by A. Jaffe

Received October 13, 1982; in revised form October 21, 1982 
